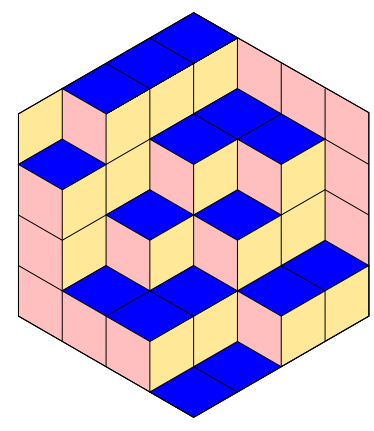

ALGEBRAIC COMBINATORICS

Yasuhide Numata \& Yuiko Yamanouchi

On the action of the toggle group of the Dynkin diagram of type $A$

Volume 5, issue 1 (2022), p. 149-161.

https://doi.org/10.5802/alco. 204

(C) The journal and the authors, 2022.

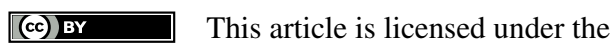

Creative Commons Attribution 4.0 International LiCEnse.

http://creativecommons.org/licenses/by/4.0/

Access to articles published by the journal Algebraic Combinatorics on the website http://alco.centre-mersenne.org/ implies agreement with the Terms of Use (http://alco.centre-mersenne.org/legal/).

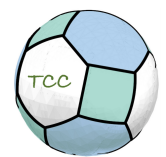

Algebraic Combinatorics is published by The Combinatorics Consortium and is a member of the Centre Mersenne for Open Scientific Publishing

www.tccpublishing.org

www.centre-mersenne.org 


\title{
On the action of the toggle group of the Dynkin diagram of type $A$
}

\author{
Yasuhide Numata \& Yuiko Yamanouchi
}

\begin{abstract}
In this article, we consider involutions, called togglings, on the set of independent sets of the Dynkin diagram of type $A$, or a path graph. We are interested in the action of the subgroup of the symmetric group of the set of independent sets generated by togglings. We show that the subgroup coincides with the symmetric group.
\end{abstract}

\section{INTRODUCTION}

In this article, we are interested in the group generated by operations called toggling. The operation, toggling, is originally introduced for the set of order ideals of a poset in Cameron-Fon-Der-Flaass [1]. Let $P$ be a finite poset, $J(P)$ the set of order ideals of $P$. For an element $p \in P$, we define the map $\tau_{p}: J(P) \rightarrow J(P)$ by

$$
\tau_{p}(I)= \begin{cases}I \cup\{p\} & (p \text { is a minimal element of } P \backslash I), \\ I \backslash\{p\} & (p \text { is a maximal element of } I), \\ I & \text { (otherwise) }\end{cases}
$$

for $I \in J(P)$. The toggle group is a subgroup of the symmetric group of $J(P)$ generated by togglings. Since we have a bijection from $J(P)$ to the set of antichains of $P$ which maps an order ideal to the maximal elements of the order ideal, we can regard toggling as an involution on the set of antichains. Moreover we can define an analogue of the toggling $\tau_{p}$ as involutions not only on antichains of a poset, but also on important classes of subsets of a poset, e.g. order ideals of a poset, chains of a poset, antichains of a poset, interval-closed sets of a certain family of posets. Moreover we can consider a toggle group on nice subsets of a finite set with a combinatorial structure, e.g. independent sets of a graph, which is the main topic in this article. The toggle groups and their orbit structures are studied from the viewpoint of dynamical algebraic combinatorics, e.g. Striker-Williams [8], Striker [7], Joseph [2], and Joseph-Roby [4].

In this article, we write $A_{n}$ to denote the Dynkin diagram of type $A_{n}$, i.e. the path graph with $n$ vertices, and consider togglings on the set of the independent sets, i.e. subsets of vertices such that no pair are adjacent in the graph, of the graph $A_{n}$. In [3], Joseph and Roby study the orbit structure and the phenomenon called

Manuscript received 31st March 2021, revised 17th November 2021, accepted 8th November 2021.

Keywords. Coxeter groups; Togglings of independent sets; Fibonacci sequence; Symmetric group; Transitive actions.

ACKNowledgements. The first author was partially supported by JSPS KAKENHI Grant Number JP18K03206. 
homomesy and related properties for the togglings. They consider the action of the product of togglings of each vertex of the graph $A_{n}$ from left to right, and a map $\chi_{i}$ defined as $\chi_{i}(I)=1$ if the independent set $I$ contains $i$-th vertices, and otherwise $\chi_{i}(I)=0$. They give a proof for Propp's conjecture that the statistics $\chi_{j}-\chi_{n+1-j}$ are 0 -mesic under the action for all $1 \leqslant j \leqslant n$. Moreover they also show that the statistics $2 \chi_{1}+\chi_{2}$ and $\chi_{n-1}+2 \chi_{n}$ are 1-mesic under the action.

In this article, we are interested in the transitivity of the action of the toggle group on the set of independent sets. For a nonempty independent set $I$, the number of vertices in the independent set $I$ is greater than the number of vertices in the resulting independent set $\tau_{v}(I)$ by the toggling with respect to a vertex $v$ in $I$. Hence we can obtain the empty independent set from $I$ by applying the product of the togglings with respect to all vertices in the independent set. It follows from this observation that the action is 1-transitive. Multiple transitivity, however, does not seem trivial. In this paper, we consider the toggle group of the independent sets of $A_{n}$, and show that the action of the toggle group is $f_{n+2}$-transitive, where $f_{n+2}$ is the $n+2$-th Fibonacci number, i.e. the number of independent sets of $A_{n}$. In other words, we show that the toggle group coincides with the symmetric group on the set of independent sets of $A_{n}$.

REmark 1.1. In [5], Macauley, McCammond and Mortveit studied asynchronous cellular automata and their "dynamics groups." Consider the binary words of length $n$ with cyclic index, i.e. the sequence $x_{0} x_{1} x_{2} \cdots x_{n-1}$ for $x_{i} \in\{0,1\}$ with indices $i \in \mathbb{Z} / n \mathbb{Z}$. For a given map $F:\{0,1\}^{3} \rightarrow\{0,1\}$, we can consider the operation to replace the $i$-th alphabet $x_{i}$ of the word $x_{0} x_{1} x_{2} \cdots x_{n-1}$ with $F\left(x_{i-1}, x_{i}, x_{i+1}\right)$ for some index $i$. Let us consider the case where the local rule $F$ is Rule 201, i.e. a map defined by

$$
F(a, b, c)= \begin{cases}1-b & (a=c=0), \\ b & \text { (otherwise) }\end{cases}
$$

In this case, the operation for each index $i$ is invertible. Hence the operations for all indices $i$ generate a group. Naturally the binary word $x_{0} x_{1} x_{2} \cdots x_{n-1}$ can be identified with the set of indices $i$ such that $x_{i}=1$. Since indices are in $\mathbb{Z} / n \mathbb{Z}$, the set can be regarded as the subset of the vertex set of the cycle with $n$ vertices. The orbit containing the binary word $0 \cdots 0$ consists of binary words $x_{0} x_{1} x_{2} \cdots x_{n-1}$ without indices $i$ such that $x_{i}=x_{i+1}=1$, corresponding to independent sets of the cycle. The group acts on the orbit as the toggle group for the cycle. Next consider the orbit containing the binary word $0 \cdots 00110$. A binary word $x_{0} x_{1} x_{2} \cdots x_{n-1}$ is in the orbit if and only if $\left(x_{n-4}, x_{n-3}, x_{n-2}, x_{n-1}\right)=(0,1,1,0)$ and $\left(x_{i}, x_{i+1}\right) \neq(1,1)$ for $i \in\{0,1, \ldots, n-6\}$. For the binary word in the orbit, the subword $x_{0} x_{1} x_{2} \cdots x_{n-5}$ corresponds to an independent set of $A_{n-4}$. The group acts on the orbit as the toggle group for $A_{n-4}$. In [5], Macauley, McCammond and Mortveit conjectured that the group acts on the orbit with $0 \cdots 0$ as the symmetric group or the alternating group on the orbit, and that the group acts on the orbit with $0 \cdots 00110$ as the symmetric group on the orbit. In [6], Salo proved the conjecture by automaton-theoretical or language-theoretical methods. Our results give another proof of the conjecture.

This article is organized as follows: In Section 2, we consider the family of the symmetric groups indexed by the Fibonacci sequence. We give systems of generators for them as Theorem 2.6. In Section 3, we recall the definition of toggling on independent sets of a graph, and we consider the toggle group for independent sets of the graph $A_{n}$. As Theorem 3.7, we state that the toggle groups for independent sets of the graph $A_{n}$ are isomorphic to the symmetric groups indexed by the Fibonacci sequence. We give proofs of main theorems in Section 4. 


\section{Systems of Generators For SyMmetric Groups indeXed By the FibONACCI SEQUENCE}

Let $\left\{f_{n}\right\}_{n=0,1, \ldots}$ be the Fibonacci sequence, i.e. the sequence of numbers defined by $f_{0}=0, f_{1}=1$, and $f_{n}=f_{n-1}+f_{n-2}$. We define $F_{n+2}$ to be the set $\left\{1,2, \ldots, f_{n+2}\right\}$. We decompose $F_{n+2}$ into $F_{n+1}$ and $\hat{F}_{n}$, where

$$
\begin{aligned}
\hat{F}_{n} & =F_{n+2} \backslash F_{n+1} \\
& =\left\{1+f_{n+1}, 2+f_{n+1}, \ldots, f_{n}+f_{n+1}=f_{n+2}\right\} \\
& =\left\{i+f_{n+1} \mid i \in F_{n}\right\} .
\end{aligned}
$$

Here we consider the family $\left\{\mathfrak{S}_{f_{n}}\right\}_{n=3,4, \ldots}$ of symmetric groups indexed by the Fibonacci sequence. For $n \leqslant m$, we regard $\mathfrak{S}_{n}$ as a subset of $\mathfrak{S}_{m}$ in the usual manner. In this article, for a set $X, \mathfrak{S}_{X}$ stands for the symmetric group on $X$. Under this notation, $\mathfrak{S}_{f_{n}}=\mathfrak{S}_{F_{n}}$.

For $n=1,2, \ldots$, we define $\hat{t}_{n} \in \mathfrak{S}_{f_{n+2}}$ to be the product

$$
\left(1, f_{n+1}+1\right)\left(2, f_{n+1}+2\right) \cdots\left(f_{n}, f_{n+1}+f_{n}\right)
$$

of $f_{n}$ transpositions. For $n \geqslant 1$, the inner automorphism by $\hat{t}_{n}$ induces the isomorphism

$$
\begin{aligned}
\mathfrak{S}_{F_{n}} & \rightarrow \mathfrak{S}_{\breve{F}_{n}} \\
t & \mapsto \hat{t}_{n} t \hat{t}_{n}^{-1} .
\end{aligned}
$$

Since $F_{n}=\left\{1,2, \ldots, f_{n}\right\}$ and $\check{F}_{n}=\left\{i+f_{n+1} \mid i \in F_{n}\right\}$, we have $F_{n} \cap \check{F}_{n}=\varnothing$, which implies that every element of $\mathfrak{S}_{F_{n}}$ commutes with every element of $\mathfrak{S}_{\breve{F}_{n}}$. For $t$ and $t^{\prime} \in \mathfrak{S}_{f_{n}}$, it follows that $t^{\prime} \in \mathfrak{S}_{f_{n}}$ commutes with $\hat{t}_{n} t \hat{t}_{n}^{-1} \in \mathfrak{S}_{\breve{F}_{n}}$, which implies

$$
t \cdot \hat{t}_{n} t \hat{t}_{n}^{-1} \cdot t^{\prime} \cdot \hat{t}_{n} t^{\prime} \hat{t}_{n}^{-1}=t \cdot t^{\prime} \cdot \hat{t}_{n} t \hat{t}_{n}^{-1} \cdot \hat{t}_{n} t^{\prime} \hat{t}_{n}^{-1}=t t^{\prime} \cdot \hat{t}_{n} t t^{\prime} \hat{t}_{n}^{-1}
$$

Hence the inner automorphism by $\hat{t}_{n}$ induces the isomorphism

$$
\begin{aligned}
\mathfrak{S}_{f_{n}} & \rightarrow \widetilde{\mathfrak{S}}_{f_{n}} \\
t & \mapsto t \cdot \hat{t}_{n} t \hat{t}_{n}^{-1},
\end{aligned}
$$

where

$$
\tilde{\mathfrak{S}}_{f_{n}}=\left\{\begin{array}{l|l}
g \in \mathfrak{S}_{f_{n+2}} & \begin{array}{c}
\forall i \leqslant f_{n}, g\left(i+f_{n+1}\right)=g(i)+f_{n+1} \\
f_{n}<\forall i \leqslant f_{n+1}, g(i)=i
\end{array} \\
\subset \mathfrak{S}_{F_{n}} \times \mathfrak{S}_{\hat{F}_{n}} \subset \mathfrak{S}_{F_{n+1}} \times \mathfrak{S}_{\hat{F}_{n}} \subset \mathfrak{S}_{F_{n+2}} .
\end{array}\right.
$$

For $1 \leqslant k \leqslant n$, we define $t_{k, n} \in \mathfrak{S}_{F_{n+2}}$ by

$$
t_{k, n}= \begin{cases}t_{k, n-1} \cdot \hat{t}_{n} t_{k, n-2} \hat{t}_{n}^{-1} & (k \leqslant n-2), \\ t_{n-1, n-1} & (k=n-1), \\ \hat{t}_{n} & (i=n),\end{cases}
$$

recursively. By definition, for $k<n, t_{k, n}$ is an element of $\mathfrak{S}_{F_{n+1}} \times \mathfrak{S}_{\hat{F}_{n}}$, and $t_{n-1, n}$ is an element of $\mathfrak{S}_{F_{n+1}}$. We define subsets $G_{n}$ and $G_{n}^{\prime}$ of $\mathfrak{S}_{F_{n+2}}$ by

$$
\begin{aligned}
& G_{n}^{\prime}=\left\{t_{k, n} \mid k \leqslant n-2\right\}, \\
& G_{n}=\left\{t_{k, n} \mid k \leqslant n\right\} .
\end{aligned}
$$

EXAmple 2.1. Consider the case where $n=1$. Since $\hat{t}_{1}$ is the transposition $(1,2) \in$ $\mathfrak{S}_{f_{3}}=\mathfrak{S}_{2}$, we have $t_{1,1}=\hat{t}_{1}=(1,2)$ and $G_{1}=\{(1,2)\}$. Hence $G_{1}$ generates $\mathfrak{S}_{2}=\mathfrak{S}_{f_{3}}$. 
EXAMPLE 2.2. In the case where $n=2, \hat{t}_{2}$ is the transposition $(1,3) \in \mathfrak{S}_{f_{4}}=\mathfrak{S}_{3}$, we have $t_{1,2}=t_{1,1}=(1,2)$ and $t_{2,2}=\hat{t}_{2}=(1,3)$. Hence $G_{2}=\{(1,2),(1,3)\}$. The set $G_{2}$ generates $\mathfrak{S}_{3}=\mathfrak{S}_{f_{4}}$.

EXAMPLE 2.3. In the case where $n=3, \hat{t}_{4}$ is the product $(1,4)(2,5) \in \mathfrak{S}_{f_{5}}=\mathfrak{S}_{5}$ of the transpositions, we have

$$
\begin{aligned}
& t_{1,3}=(1,2) \cdot(4,5), \\
& t_{2,3}=t_{2,2}=(1,3), \\
& t_{3,3}=\hat{t}_{3}=(1,4)(2,5) .
\end{aligned}
$$

Hence

$$
\begin{aligned}
G_{3} & =\{(1,2)(4,5),(1,3),(1,4)(2,5)\}, \\
G_{3}^{\prime} & =\{(1,2)(4,5)\} .
\end{aligned}
$$

Since

$$
\begin{aligned}
& \tilde{\mathfrak{S}}_{f_{3}}=\left\{\begin{array}{l|l}
g \in \mathfrak{S}_{f_{5}} & \begin{array}{c}
\forall i \leqslant f_{3}, g\left(i+f_{4}\right)=g(i)+f_{4} \\
f_{3}<\forall i \leqslant f_{4}, g(i)=i
\end{array}
\end{array}\right\} \\
& =\left\{g \in \mathfrak{S}_{f_{5}} \mid g(1+3)=g(1)+3, g(2+3)=g(2)+3, g(3)=3\right\} \text {, }
\end{aligned}
$$

the group $\tilde{\mathfrak{S}}_{f_{3}}$ is generated by $\{(1,2)(4,5)\}$. It follows from direct calculation that

$$
\begin{aligned}
& (1,3)=t_{2,3}, \\
& (2,3)=t_{1,3}(1,3) t_{1,3}^{-1}, \\
& (4,3)=t_{3,3}(1,3) t_{3,3}^{-1}, \\
& (5,3)=t_{1,3}(4,3) t_{1,3}^{-1} .
\end{aligned}
$$

Hence $G_{3}$ generates $\mathfrak{S}_{5}=\mathfrak{S}_{f_{5}}$.

EXAmple 2.4. Consider the case where $n=4$. In this case, $\hat{t}_{4}=(1,6)(2,7)(3,8) \in$ $\mathfrak{S}_{f_{6}}=\mathfrak{S}_{8}$ of the transpositions. Hence

$$
\begin{aligned}
& t_{1,4}=(1,2)(4,5) \cdot(6,7), \\
& t_{2,4}=(1,3) \cdot(6,8), \\
& t_{3,4}=t_{3,3}=(1,4)(2,5), \\
& t_{4,4}=\hat{t}_{4}=(1,6)(2,7)(3,8) .
\end{aligned}
$$

Hence

$$
\begin{aligned}
G_{4} & =\{(1,2)(4,5)(6,7),(1,3)(6,8),(1,4)(2,5),(1,6)(2,7)(3,8)\}, \\
G_{4}^{\prime} & =\{(1,2)(4,5)(6,7),(1,3)(6,8)\} .
\end{aligned}
$$

We show the following theorems by induction on $n$ in Subsection 4.1.

THEOREM 2.5. For $n=3,4, \ldots$, the set $G_{n}^{\prime}$ generates the group $\tilde{\mathfrak{S}}_{f_{n}}$. Hence the group $\left\langle G_{n}^{\prime}\right\rangle$ generated by $G_{n}^{\prime}$ is isomorphic to $\mathfrak{S}_{f_{n}}$.

TheOREM 2.6. For $n=1,2, \ldots$, the set $G_{n}$ generates the $f_{n+2}$-th symmetric group $\mathfrak{S}_{f_{n+2}}$. 


\section{The toggle Group for independent Sets of the Dynkin Diagram OF TYPE $A$}

In this article, we consider simple graphs. We regard a graph as the pair $(V, E)$ of the set $V$ of vertices and the set $E$ of edges. We also regard an edge as a subset of $V$ of size two. An independent set of a graph is a subset $I$ of the vertex set $V$ of the graph satisfying

$$
u, v \in I \Longrightarrow\{u, v\} \notin E .
$$

Roughly speaking, an independent set of a graph is a subset of vertices such that no pair is adjacent in $(V, E)$.

Let $\mathcal{I}$ be the set of independent sets of a graph $(V, E)$. For a vertex $v \in V$, we define the map $\tau_{v}$ by

$$
\begin{aligned}
\tau_{v}: \mathcal{I} & \rightarrow \mathcal{I} \\
I & \mapsto \begin{cases}I \backslash\{v\} & (v \in I), \\
I \cup\{v\} & (v \notin I \text { and } I \cup\{v\} \in \mathcal{I}), \\
I & \text { (otherwise) } .\end{cases}
\end{aligned}
$$

By definition, the map $\tau_{v}$ is an involution on $\mathcal{I}$. Hence $\tau_{v}$ is an element of the symmetric group $\mathfrak{S}_{\mathcal{I}}$ on $\mathcal{I}$. We call the map $\tau_{v}$ the toggling on $\mathcal{I}$ with respect to $v$. We also call the subgroup generated by all togglings the toggle group of the graph $(V, E)$.

REMARK 3.1. Let $\mathcal{I}$ be the set of independent sets of a graph $(V, E)$. For a vertex $v \in V$, we can also describe the toggling $\tau_{v}$ as follows: We define $N_{v}(V, E)$ to be the set of neighbours of $v$, i.e. $\{u \mid\{u, v\} \in E\}$. Let $\mathcal{I}^{\prime}=\left\{I \in \mathcal{I} \mid I \cap N_{v}(V, E) \neq \varnothing\right\}$. The toggling $\tau_{v}$ acts on $\mathcal{I}^{\prime}$ trivially. Let

$$
\begin{aligned}
& V^{\prime}=V \backslash\left(\{v\} \cup N_{v}(V, E)\right), \\
& E^{\prime}=\left\{\{u, w\} \in E \mid u, w \in V^{\prime}\right\} .
\end{aligned}
$$

The graph $\left(V^{\prime}, E^{\prime}\right)$ is the induced subgraph of the graph $(V, E)$ with respect to all vertices except $v$ and its neighbours. Let $\mathcal{I}^{\prime \prime}$ be the set of independent sets of $\left(V^{\prime}, E^{\prime}\right)$. For $I \in \mathcal{I}^{\prime \prime}, I$ and $I \cup\{v\}$ are independent sets of $(V, E)$. Then we have $\tau_{v}(I)=I \cup\{v\}$ and $\tau_{v}(I \cup\{v\})=I$ for $I \in \mathcal{I}^{\prime \prime}$. Since $\mathcal{I}=\mathcal{I}^{\prime} \cup \mathcal{I}^{\prime \prime} \cup \mathcal{I}^{\prime \prime \prime}$ for $\mathcal{I}^{\prime \prime \prime}=\left\{I \cup\{v\} \mid I \in \mathcal{I}^{\prime \prime}\right\}$, the toggling $\tau_{v}$ is the involution on $\mathcal{I}$ defined by

$$
\begin{cases}\tau(I)=\tau(I) & \left(I \in \mathcal{I}^{\prime}\right), \\ \tau(I)=\tau(I \cup\{v\}) & \left(I \in \mathcal{I}^{\prime \prime}\right), \\ \tau(I \cup\{v\})=\tau(I) & \left(I \in \mathcal{I}^{\prime \prime}\right) .\end{cases}
$$

The toggling $\tau_{v} \in \mathfrak{S}_{\mathcal{I}}$ is the product of transpositions swapping $I$ and $I \cup\{v\}$. Hence, the toggling $\tau_{v} \in \mathfrak{S}_{\mathcal{I}}$ is an even permutation if and only if \# $\# \mathcal{I}^{\prime}$ is even.

REMARK 3.2. The toggle group acts on the set $\mathcal{I}$ of independent sets naturally. Moreover, for an independent set $I=\left\{v_{1}, v_{2}, \ldots, v_{l}\right\}$ of size $l$, we have

$$
\tau_{v_{1}} \circ \tau_{v_{2}} \circ \cdots \circ \tau_{v_{l}}(I)=\varnothing .
$$

We can obtain the empty set from any independent set by applying some togglings. Hence the group generated by all togglings of a graph acts transitively on the set of independent sets of the graph. In other words, the action of the toggle group is 1-transitive.

We give some small examples. First we consider cycles. The toggle group of some cycle is a proper subgroup of the symmetric group on the set of independent sets. 
ExAmple $3.3\left(\tilde{A}_{3}\right)$. Consider the cycle $(V, E)$ such that

$$
V=\{0,1,2,3\}, \quad E=\{\{0,1\},\{1,2\},\{2,3\},\{3,0\}\} .
$$

and the set $\mathcal{I}$ of its independent sets. Consider the toggling $\tau_{0}$ for the vertex 0 . The induced subgraph with respect to all vertices except 0 and neighbours of 0 is $(\{2\}, \varnothing)$. The set of its independent sets is $\{\varnothing,\{2\}\}$, consisting of two elements. Hence $\tau_{0} \in \mathfrak{S}_{\mathcal{I}}$ is an even permutation. Similarly, we can show that $\tau_{v} \in \mathfrak{S}_{\mathcal{I}}$ is an even permutation for each vertex $v \in V$. Hence we have the toggle group is a subgroup of the alternating group on $\mathcal{I}$, which is a proper subgroup of the symmetric group $\mathfrak{S}_{\mathcal{I}}$.

Next we consider trees. For the tree in Example 3.4, the toggle group equals the symmetric group on the set of independent sets. For the tree in Example 3.5, the toggle group is a proper subgroup of the symmetric group on the set of independent sets.

ExAmple $3.4\left(D_{4}\right)$. Consider the tree $(V, E)$ such that

$$
V=\{1,2,3,4\}, \quad E=\{\{1,3\},\{2,3\},\{3,4\}\} .
$$

The set $\mathcal{I}$ of independent sets of the tree consists of the following:

$$
\begin{aligned}
& I_{1}=\varnothing \quad I_{2}=\{1\} \quad I_{3}=\{2\} \quad I_{4}=\{1,2\} \\
& I_{5}=\{4\} \quad I_{6}=\{1,4\} \quad I_{7}=\{2,4\} \quad I_{8}=\{1,2,4\} \quad I_{9}=\{3\} .
\end{aligned}
$$

We regard $\mathcal{I}$ as $\{1,2, \ldots, 9\}$ by identifying $I_{k}$ with $k$. Then togglings are the product of the transpositions as follows:

$$
\begin{array}{ll}
\tau_{1}=(1,2)(3,4)(5,6)(7,8) & \tau_{2}=(1,3)(2,4)(5,7)(6,8) \\
\tau_{4}=(1,5)(2,6)(3,7)(4,8) & \tau_{3}=(1,9) .
\end{array}
$$

Let

$$
\begin{array}{llll}
\sigma_{2}=\tau_{1}, & \sigma_{3}=\tau_{2}, & \sigma_{4}=\tau_{2} \tau_{1}, & \sigma_{5}=\tau_{4}, \\
\sigma_{6}=\tau_{4} \tau_{1}, & \sigma_{7}=\tau_{4} \tau_{2}, & \sigma_{8}=\tau_{4} \tau_{2} \tau_{1} . &
\end{array}
$$

Then we have $\sigma_{k}(1)=k$ and $\sigma_{k}(9)$. Hence we have

$$
\sigma_{k} \tau_{3} \sigma_{k}^{-1}=\left(\sigma_{k}(1), \sigma_{k}(9)\right)=(k, 9) .
$$

Since we have $(1,9),(2,9), \ldots,(8,9)$ in the toggle group, the toggle group is the symmetric group $\mathfrak{S}_{\mathcal{I}}$.

ExAMPLE $3.5\left(\tilde{D}_{5}\right)$. Consider the tree $(V, E)$ such that

$$
V=\{0,1,2,3,4,5\}, \quad E=\{\{1,3\},\{2,3\},\{3,4\},\{4,5\},\{4,0\}\},
$$

and the set $\mathcal{I}$ of its independent sets. Consider the toggling $\tau_{3}$ for the vertex 3 . The induced subgraph with respect to all vertices except 3 and neighbours of 3 is the disjoint union of the graphs $(\{0\}, \varnothing)$ and $(\{5\}, \varnothing)$. The number of the independent sets of the disjoint union of graphs is the product of the number of the independent sets of each graph. Since the number of the independent sets of $(\{0\}, \varnothing)$ is two, the number of the independent sets of the induced graph is even. Hence $\tau_{3} \in \mathfrak{S}_{\mathcal{I}}$ is even. Similarly, we have $\tau_{4}$ is even. Next consider the toggling $\tau_{1}$ for the vertex 1. The induced subgraph with respect to all vertices except 1 and neighbours of 1 is the disjoint union of the graphs $(\{2\}, \varnothing)$ and $(\{0,4,5\},\{\{0,4\},\{4,5\}\})$. Hence we have the number of independent sets of the induced subgraph is even, which implies $\tau_{1}$ is even. Similarly, it follows that $\tau_{v}$ is even for $v \in\{0,2,5\}$. Hence the toggle group is a subgroup of the alternating group on $\mathcal{I}$, which is a proper subgroup of $\mathfrak{S}_{\mathcal{I}}$. 
Let $A_{n}=\left(V_{n}, E_{n}\right)$ be the graph such that

$$
\begin{aligned}
V_{n} & =\{1, \ldots, n\}, \\
E_{n} & =\{\{1,2\},\{2,3\} \ldots,\{n-1, n\}\},
\end{aligned}
$$

i.e. the Dynkin diagram of type $A_{n}$. Let $\mathcal{I}_{n}$ be the set of independent sets of $A_{n}$. In this article, we are interested in the togglings of the graph $A_{n}$.

For $1 \leqslant k \leqslant n$, we define $\tau_{k, n}$ to be the toggling $\tau_{k}$ on $\mathcal{I}_{n}$ with respect to $k \in V_{n}$, i.e.

$$
\begin{aligned}
\tau_{k, n}: \mathcal{I}_{n} & \rightarrow \mathcal{I}_{n} \\
I & \mapsto \begin{cases}I \backslash\{k\} & (k \in I), \\
I \cup\{k\} & (k-1, k, k+1 \notin I), \\
I & \text { (otherwise). }\end{cases}
\end{aligned}
$$

We also define define $\Gamma_{n}$ to be the group generated by togglings

$$
\left\{\tau_{1, n}, \tau_{2, n}, \ldots, \tau_{n, n}\right\} \text {. }
$$

We call this group the toggle group of $A_{n}$. By definition the toggle group $\Gamma_{n}$ of $A_{n}$ acts on the set $\mathcal{I}_{n}$ of independent sets on $A_{n+1}$, naturally. In the other words, the toggle group $\Gamma_{n}$ is a subgroup of the symmetric group $\mathfrak{S}_{\mathcal{I}_{n}}$ of $\mathcal{I}_{n}$.

REMARK 3.6. The togglings $\tau_{k, n}$ for $1 \leqslant k \leqslant n$ satisfy the following relations:

$$
\begin{aligned}
\tau_{k, n}^{2} & =\mathrm{id} . \\
\tau_{k, n} \tau_{k^{\prime}, n} & =\tau_{k^{\prime}, n} \tau_{k, n} \quad\left(\left|k-k^{\prime}\right|>1\right) . \\
\left(\tau_{k, n} \tau_{k+1, n}\right)^{6} & =\mathrm{id} .
\end{aligned}
$$

Hence the toggle group $\Gamma_{n}$ is a finite quotient group of the Coxeter group with respect to

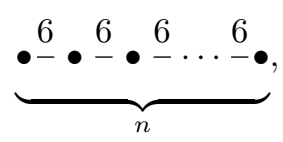

which is infinite if $n>2$.

We will show the following theorem in Subsection 4.2.

THEOREM 3.7. For $n=1,2, \ldots$, the toggle group $\Gamma_{n}$ of $A_{n}$ is the symmetric group $\mathfrak{S}_{\mathcal{I}_{n}}$ of the set $\mathcal{I}_{n}$ of independent sets of $A_{n}$. Hence the toggle group $\Gamma_{n}$ of $A_{n}$ is isomorphic to the $f_{n+2}$-th symmetric group $\mathfrak{S}_{f_{n+2}}$.

Corollary 3.8. For $n=1,2, \ldots$, the action of the toggle group $\Gamma_{n}$ of $A_{n+1}$ on $\mathcal{I}_{n}$ is $f_{n+2}$-transitive.

\section{Proof of Main Theorems}

4.1. Theorems 2.5 AND 2.6. Here we show these Theorems 2.5 and 2.6 by induction on $n$. To show theorems, we show some lemmas.

LEMmA 4.1. If $n \geqslant 3$ and $G_{n-2}$ generates $\mathfrak{S}_{f_{n}}$, then $G_{n}^{\prime}$ generates $\tilde{\mathfrak{S}}_{f_{n}}$.

Proof. Consider the isomorphism

$$
\begin{aligned}
\varphi: \mathfrak{S}_{f_{n}} & \rightarrow \tilde{\mathfrak{S}}_{f_{n}} \\
t & \mapsto t \cdot \hat{t}_{n} t \hat{t}_{n}^{-1} .
\end{aligned}
$$


By definition, for $k \leqslant n-2, t_{k, n}$ is the image $\varphi\left(t_{k, n-2}\right)$ of $t_{k, n-2}$. Hence we have

$$
\begin{aligned}
\left\langle G_{n}^{\prime}\right\rangle & =\left\langle\varphi\left(t_{1, n-2}\right), \varphi\left(t_{2, n-2}\right), \ldots, \varphi\left(t_{n-2, n-2}\right)\right\rangle \\
& =\varphi\left(\left\langle t_{1, n-2}, t_{2, n-2}, \ldots, t_{n-2, n-2}\right\rangle\right) \\
& =\varphi\left(\left\langle G_{n-2}\right\rangle\right) .
\end{aligned}
$$

Since $G_{n-2}$ generates $\mathfrak{S}_{f_{n}}$, we have $\left\langle G_{n}^{\prime}\right\rangle=\varphi\left(\mathfrak{S}_{f_{n}}\right)=\tilde{\mathfrak{S}}_{f_{n}}$.

LEMMA 4.2. If $n \geqslant 4$ and $G_{n}^{\prime}$ generates $\tilde{\mathfrak{S}}_{f_{n}}$, then the group $\left\langle G_{n}\right\rangle$ generated by $G_{n}$ contains cyclic permutations $(i, i+1, i+2)$ for $1 \leqslant i \leqslant f_{n+2}-2$.

Proof. We decompose $F_{n+2}$ into three subsets

$$
\begin{aligned}
F_{n} & =\left\{1,2, \ldots, f_{n}\right\}, \\
\hat{F}_{n-1} & =\left\{i+f_{n} \mid i \in F_{n-1}\right\}, \\
\hat{F}_{n} & =\left\{i+f_{n+1} \mid i \in F_{n}\right\} .
\end{aligned}
$$

First we show that $(1,2,3) \in\left\langle G_{n}\right\rangle$. Since $n \geqslant 4, f_{n}>f_{3}=2$. Since $\tilde{\mathfrak{S}}_{f_{n}}=\left\langle G_{n}^{\prime}\right\rangle \subset$ $\left\langle G_{n}\right\rangle$, the group $\left\langle G_{n}\right\rangle$ contains the following elements:

$$
\begin{aligned}
& (1,2) \hat{t}_{n}(1,2) \hat{t}_{n}^{-1}=(1,2)\left(f_{n+1}+1, f_{n+1}+2\right), \\
& (2,3) \hat{t}_{n}(2,3) \hat{t}_{n}^{-1}=(2,3)\left(f_{n+1}+2, f_{n+1}+3\right) .
\end{aligned}
$$

Since $t_{n-1, n}=\hat{t}_{n-1} \in G_{n}$, we have

$$
\hat{t}_{n-1}(1,2)\left(f_{n+1}+1, f_{n+1}+2\right) \hat{t}_{n-1}^{-1} \in\left\langle G_{n}\right\rangle \text {. }
$$

Since $\hat{t}_{n-1} \in \mathfrak{S}_{F_{n+1}}$,

$$
\begin{aligned}
\hat{t}_{n-1}(1,2)\left(f_{n+1}+1, f_{n+1}+2\right) \hat{t}_{n-1}^{-1} & =\hat{t}_{n-1}(1,2) \hat{t}_{n-1}^{-1} \cdot\left(f_{n+1}+1, f_{n+1}+2\right) \\
& =\left(f_{n}+1, f_{n}+2\right)\left(f_{n+1}+1, f_{n+1}+2\right) .
\end{aligned}
$$

Hence $\left\langle G_{n}\right\rangle$ contains

$$
\begin{aligned}
\left(f_{n}+1, f_{n}+2\right) & \left(f_{n+1}+1, f_{n+1}+2\right) \cdot(2,3)\left(f_{n+1}+2, f_{n+1}+3\right) \\
= & (2,3) \cdot\left(f_{n}+1, f_{n}+2\right) \cdot\left(f_{n+1}+1, f_{n+1}+2\right)\left(f_{n+1}+2, f_{n+1}+3\right) \\
= & (2,3) \cdot\left(f_{n}+1, f_{n}+2\right) \cdot\left(f_{n+1}+1, f_{n+1}+2, f_{n+1}+3\right) .
\end{aligned}
$$

Hence $\left\langle G_{n}\right\rangle$ contains the square

$$
\begin{aligned}
\left((2,3) \cdot\left(f_{n}+1, f_{n}+2\right)\right. & \left.\cdot\left(f_{n+1}+1, f_{n+1}+2, f_{n+1}+3\right)\right)^{2} \\
= & (2,3)^{2} \cdot\left(f_{n}+1, f_{n}+2\right)^{2} \cdot\left(f_{n+1}+1, f_{n+1}+2, f_{n+1}+3\right)^{2} \\
= & \left(f_{n+1}+1, f_{n+1}+3, f_{n+1}+2\right)
\end{aligned}
$$

of the element. Since $\hat{t}_{n}\left(f_{n+1}+1, f_{n+1}+3, f_{n+1}+2\right) \hat{t}_{n}^{-1}=(1,3,2),\left\langle G_{n}\right\rangle$ contains cyclic permutations $(1,3,2)$ and $(1,2,3)$.

Next we show that $\left\langle G_{n}\right\rangle$ contains the cyclic permutation $(i, j, k)$ for $i, j, k \in F_{n}$. Fix an element $\sigma \in \mathfrak{S}_{F_{n}}$ such that $\sigma(1)=i, \sigma(2)=j, \sigma(3)=k$. Since $\widetilde{\mathfrak{S}}_{f_{n}}=\left\langle G_{n}^{\prime}\right\rangle \subset\left\langle G_{n}\right\rangle$, $\left\langle G_{n}\right\rangle$ contains $\tilde{\sigma}=\sigma \hat{t}_{n} \sigma \hat{t}_{n}^{-1} \in \tilde{\mathfrak{S}}_{f_{n}}$. Hence $\left\langle G_{n}\right\rangle$ contains

$$
\tilde{\sigma}(1,2,3) \tilde{\sigma}^{-1}=(\tilde{\sigma}(1), \tilde{\sigma}(2), \tilde{\sigma}(3))=(\sigma(1), \sigma(2), \sigma(3))=(i, j, k) .
$$

Next we show that $\left\langle G_{n}\right\rangle$ contains the cyclic permutation $(i, j, k)$ for $i, j, k \in \hat{F}_{n-1}=$ $\left\{i^{\prime}+f_{n} \mid i^{\prime} \in F_{n-1}\right\}$. Since $F_{n-1}$ contains $i-f_{n}, j-f_{n}$ and $k-f_{n}$ for $i, j, k \in \hat{F}_{n-1}$, 
$\left\langle G_{n}\right\rangle$ contains $\left(i-f_{n}, j-f_{n}, k-f_{n}\right)$. Hence $\left\langle G_{n}\right\rangle$ contains

$$
\begin{aligned}
\hat{t}_{n-1}\left(i-f_{n}, j-f_{n}, k-f_{n}\right) \hat{t}_{n-1}^{-1} & \left.=\left(\hat{t}_{n-1}\left(i-f_{n}\right), \hat{t}_{n-1}\left(j-f_{n}\right)\right) \hat{t}_{n-1}\left(k-f_{n}\right)\right) \\
& =(i, j, k) .
\end{aligned}
$$

Next we show that $\left\langle G_{n}\right\rangle$ contains the cyclic permutation $(i, j, k)$ for $i, j, k \in \hat{F}_{n}=$ $\left\{i+f_{n+1} \mid i \in F_{n}\right\}$. Since $F_{n}$ contains $i-f_{n+1}, j-f_{n+1}$ and $k-f_{n+1}$ for $i, j, k \in \hat{F}_{n}$, $\left\langle G_{n}\right\rangle$ contains $\left(i-f_{n+1}, j-f_{n+1}, k-f_{n+1}\right)$. Hence $\left\langle G_{n}\right\rangle$ contains

$$
\begin{aligned}
\hat{t}_{n}\left(i-f_{n+1}, j-f_{n+1}, k-f_{n+1}\right) \hat{t}_{n}^{-1} & \left.=\left(\hat{t}_{n}\left(i-f_{n+1}\right), \hat{t}_{n-1}\left(j-f_{n+1}\right)\right) \hat{t}_{n}\left(k-f_{n+1}\right)\right) \\
& =(i, j, k) .
\end{aligned}
$$

Next $\left\langle G_{n}\right\rangle$ contains the cyclic permutation $(i, j, k)$ for $i \in F_{n}$ and $j, k \in \hat{F}_{n-1}=$ $\left\{i^{\prime}+f_{n} \mid i^{\prime} \in F_{n-1}\right\}$. For $j, k \in \hat{F}_{n-1}$, we have $j-f_{n}$ and $k-f_{n} \in F_{n-1} \subset F_{n}$. Since $f_{n} \in F_{n} \backslash F_{n-1},\left\langle G_{n}\right\rangle$ contains the cyclic permutation $\left(f_{n}, j-f_{n}, k-f_{n}\right)$. Hence $\left\langle G_{n}\right\rangle$ contains

$$
\begin{aligned}
\hat{t}_{n-1}\left(f_{n}, j-f_{n}, k-f_{n}\right) \hat{t}_{n-1}^{-1} & =\left(\hat{t}_{n-1}\left(f_{n}\right), \hat{t}_{n-1}\left(j-f_{n}\right), \hat{t}_{n-1}\left(k-f_{n}\right)\right) \\
& =\left(f_{n}, j, k\right) .
\end{aligned}
$$

Since $\tilde{\mathfrak{S}}_{f_{n}}=\left\langle G_{n}^{\prime}\right\rangle \subset\left\langle G_{n}\right\rangle,\left\langle G_{n}\right\rangle$ contains

$$
\tilde{\sigma}=\left(i, f_{n}\right) \hat{t}_{n}\left(i, f_{n}\right) \hat{t}_{n}^{-1}=\left(i, f_{n}\right)\left(f_{n+1}+i, f_{n+1}+f_{n}\right)
$$

for $i<f_{n}$. Hence $\left\langle G_{n}\right\rangle$ contains

$$
\tilde{\sigma}\left(f_{n}, j, k\right) \tilde{\sigma}^{-1}=\left(\tilde{\sigma}\left(f_{n}\right), \tilde{\sigma}(j), \tilde{\sigma}(k)\right)=(i, j, k) .
$$

Next $\left\langle G_{n}\right\rangle$ contains the cyclic permutation $(i, j, k)$ for

$$
i, j \in \hat{F}_{n-1}=\left\{i^{\prime}+f_{n} \mid i^{\prime} \in F_{n-1}\right\} \text { and } k \in \hat{F}_{n}=\left\{i+f_{n+1} \mid i \in F_{n}\right\} .
$$

Since $k-f_{n+1} \in F_{n}$ for $k \in \hat{F}_{n},\left\langle G_{n}\right\rangle$ contains the cyclic permutation $\left(k-f_{n+1}, i, j\right)$. Hence $\left\langle G_{n}\right\rangle$ contains

$$
\begin{aligned}
\hat{t}_{n}\left(k-f_{n+1}, i, j\right) \hat{t}_{n}^{-1} & =\left(\hat{t}_{n}\left(k-f_{n+1}\right), \hat{t}_{n}(i), \hat{t}_{n}(j)\right) \\
& =(k, i, j)=(i, j, k) .
\end{aligned}
$$

Next $\left\langle G_{n}\right\rangle$ contains the cyclic permutation $(i, j, k)$ for $i, j \in F_{n}$ and $k \in \hat{F}_{n-1}=$ $\left\{i^{\prime}+f_{n} \mid i^{\prime} \in F_{n-1}\right\}$. Since $F_{n-1}$ contains $k-f_{n}$ for $k \in \hat{F}_{n-1}$ and $\hat{F}_{n-1}$ contains $f_{n}+1, f_{n}+2,\left\langle G_{n}\right\rangle$ contains $\left(k-f_{n}, f_{n}+1, f_{n}+2\right)$. Hence $\left\langle G_{n}\right\rangle$ contains

$$
\begin{aligned}
\hat{t}_{n-1}\left(k-f_{n}, f_{n}+1, f_{n}+2\right) \hat{t}_{n-1}^{-1} & =\left(\hat{t}_{n-1}\left(k-f_{n}\right), \hat{t}_{n-1}\left(f_{n}+1\right), \hat{t}_{n-1}\left(f_{n}+2\right)\right) \\
& =(k, 1,2) .
\end{aligned}
$$

Fix an element $\sigma \in \mathfrak{S}_{f_{n}}$ such that $\sigma(1)=i$ and $\sigma(2)=j$. Since $\tilde{\mathfrak{S}}_{f_{n}}=\left\langle G_{n}^{\prime}\right\rangle \subset\left\langle G_{n}\right\rangle$, $\left\langle G_{n}\right\rangle$ contains $\tilde{\sigma}=\sigma \hat{t}_{n} \sigma \hat{t}_{n}^{-1}$. Hence $\left\langle G_{n}\right\rangle$ contains

$$
\tilde{\sigma}(k, 1,2) \tilde{\sigma}^{-1}=(\tilde{\sigma}(k), \tilde{\sigma}(1), \tilde{\sigma}(2))=(k, i, j)=(i, j, k) .
$$

Next $\left\langle G_{n}\right\rangle$ contains the cyclic permutation $(i, j, k)$ for

$$
i \in \hat{F}_{n-1}=\left\{i^{\prime}+f_{n} \mid i^{\prime} \in F_{n-1}\right\} \text { and } j, k \in \hat{F}_{n}=\left\{i+f_{n+1} \mid i \in F_{n}\right\} .
$$

Since $F_{n}$ contains $j-f_{n+1} k-f_{n+1}$ for $j, k \in \hat{F}_{n},\left\langle G_{n}\right\rangle$ contains the cyclic permutation $\left(j-f_{n+1}, k-f_{n+1}, i\right)$. Hence $\left\langle G_{n}\right\rangle$ contains

$$
\begin{aligned}
\hat{t}_{n}\left(j-f_{n+1}, i-f_{n+1}, i\right) \hat{t}_{n}^{-1} & =\left(\hat{t}_{n}\left(j-f_{n+1}\right), \hat{t}_{n}\left(k-f_{n+1}\right), \hat{t}_{n}(i)\right) \\
& =(j, k, i)=(i, j, k) .
\end{aligned}
$$

Since $n \geqslant 4, \# \hat{F}_{n-1}=f_{n-1} \geqslant f_{3}=2$. Hence we have $(i, i+1, i+2) \in\langle G\rangle$ for $1 \leqslant i \leqslant f_{n+2}-2$. 
LeMmA 4.3. If $n \geqslant 4$ and $G_{n}^{\prime}$ generates $\tilde{\mathfrak{S}}_{f_{n}}$, then $G_{n}$ generates $\mathfrak{S}_{F_{n+2}}$.

Proof. Since $\left\langle G_{n}\right\rangle$ contains cyclic permutations $(i, i+1, i+2)$ for $1 \leqslant i \leqslant f_{n+2}-2$ by Lemma $4.2,\left\langle G_{n}\right\rangle$ contains the $f_{n+2}$-th alternating group $\mathfrak{A}_{f_{n+2}}$. Since either $f_{n}$ or $f_{n-1}$ is an odd number, the alternating group $\mathfrak{A}_{f_{n+2}}$ does not contain either $t_{n, n}=\hat{t}_{n}$ or $t_{n, n-1}=\hat{t}_{n-1}$. Since the alternating group $\mathfrak{A}_{f_{n+2}}$ is a subgroup of $\mathfrak{S}_{f_{n+2}}$ of index 2 , the subgroup $\left\langle G_{n}\right\rangle$ coincide with $\mathfrak{S}_{f_{n+2}}$.

By Examples 2.1, 2.2 and 2.3, we can show the base case, i.e. the case where $n=1,2,3$. Moreover we have Lemmas 4.1 and 4.3 for induction step. Hence we have Theorems 2.5 and 2.6 .

4.2. Proof of Theorem 3.7. To show Theorem 3.7, we give a bijection between $\mathcal{I}_{n}$ and $F_{n+2}$, and translate togglings to permutations in $\mathfrak{S}_{f_{n+2}}$.

We can decompose the set $\mathcal{I}_{n}$ of independent set of the graph $A_{n}$ into the following two subsets:

$$
\begin{aligned}
& \left\{I \in \mathcal{I}_{n} \mid n \notin I\right\}, \\
& \left\{I \in \mathcal{I}_{n} \mid n \in I\right\} .
\end{aligned}
$$

It is easy to see that $\left\{I \in \mathcal{I}_{n} \mid n \notin I\right\}=\mathcal{I}_{n-1}$. We define $\hat{\mathcal{I}}_{n-2}$ to be the set $\left\{I \in \mathcal{I}_{n} \mid n \in I\right\}$. For $I \in \mathcal{I}_{n}$, by definition, we have $n-1 \notin I$ if $n \in I$. Hence we have the bijection $\varphi_{n-2}$ from $\hat{\mathcal{I}}_{n-2}$ to $\mathcal{I}_{n-2}$ by removing the vertex $n$, i.e. the map defined by

$$
\begin{aligned}
\varphi_{n-2}: \hat{\mathcal{I}}_{n-2} & \rightarrow \mathcal{I}_{n-2} \\
I & \mapsto I \backslash\{n\} .
\end{aligned}
$$

Hence we have the recurrence relation $\# \mathcal{I}_{n}=\# \mathcal{I}_{n-1}+\# \mathcal{I}_{n-2}$. Since $\# \mathcal{I}_{1}=2$ and $\# \mathcal{I}_{2}=3$, the number $\# \mathcal{I}_{n}$ of independent sets of $A_{n}$ is equal to the $(n+2)$-th Fibonacci number $f_{n+2}$.

Since the number of $\mathcal{I}_{n}$ is $f_{n+2}=\# F_{n+2}$, the elements of $\mathcal{I}_{n}$ can be indexed by $F_{n+2}$. We give the index $\iota_{n}(I)$ of an independent set $I$ in $\mathcal{I}_{n}$ in the following manner: In the case where $n=1$, we define

$$
\iota_{1}(I)= \begin{cases}1 & (I=\varnothing), \\ 2 & (I=\{1\}) .\end{cases}
$$

In the case where $n=2$, we define

$$
\iota_{1}(I)= \begin{cases}1 & (I=\varnothing), \\ 2 & (I=\{1\}), \\ 3 & (I=\{2\}) .\end{cases}
$$

In the case where $n>2$, we define

$$
\iota_{n}(I)= \begin{cases}\iota_{n-1}(I) & \left(I \in \mathcal{I}_{n-1}\right), \\ \iota_{n-2}\left(\varphi_{n-2}(I)\right)+f_{n+1} & \left(I \in \hat{\mathcal{I}}_{n-2}\right),\end{cases}
$$

recursively. By definition, the index of an independent set in $\mathcal{I}_{n-1}$ is in $F_{n+1}$. The index of an independent set in $\hat{\mathcal{I}}_{n-2}$, corresponding to $\mathcal{I}_{n-2}$, is in $\hat{F}_{n}$. Moreover, if $I \in \mathcal{I}_{n}$ satisfies $\iota_{n}(I) \leqslant f_{k+2}$, then $I \in \mathcal{I}_{k}$. We also have

$$
\iota_{n}(I \cup\{n\})=\iota_{n-2}(I)+f_{n+1}=\iota_{n-1}(I)+f_{n+1}=\iota_{n}(I)+f_{n+1}
$$

for $I \in \mathcal{I}_{n-2}$. 
EXAMPLE 4.4. For $n=1,2,3,4$, independent sets in $I_{n}$ are indexed as in the Figure 1.

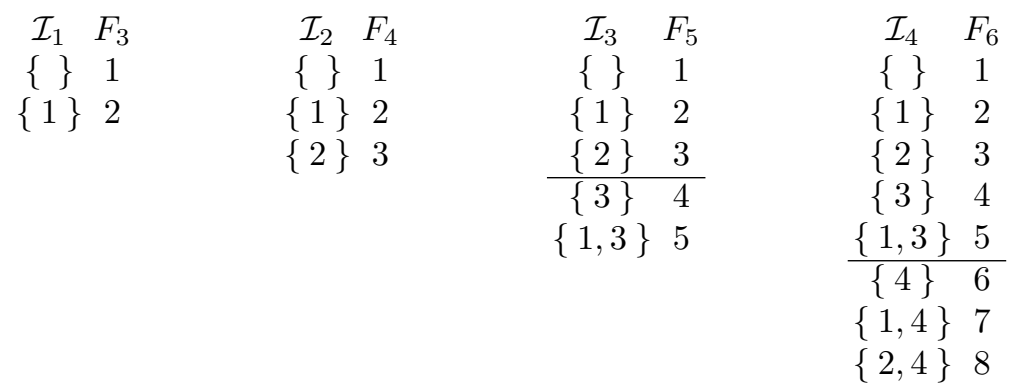

FiguRE $1 . \iota_{n}(I)$ for $n=1,2,3,4$

EXAMPLE 4.5. It follows from direct calculation that

$$
\begin{aligned}
\iota_{1}\left(\tau_{1,1}(\{\})\right) & =\iota_{1}(\{1\})=2, \\
t_{1,1}\left(\iota_{1}(\{\})\right) & =t_{1,1}(1)=(1,2)(1)=2, \\
\iota_{1}\left(\tau_{1,1}(\{1\})\right) & =\iota_{1}(\{\})=1, \\
t_{1,1}\left(\iota_{1}(\{1\})\right) & =t_{1,1}(2)=(1,2)(2)=1 .
\end{aligned}
$$

Hence

for $I \in \mathcal{I}_{1}$.

$$
\iota_{1}\left(\tau_{1,1}(I)\right)=t_{1,1}\left(\iota_{n}(I)\right)
$$

EXAMPLE 4.6. It follows from direct calculation that

$$
\begin{aligned}
\iota_{2}\left(\tau_{1,2}(\{\})\right) & =\iota_{2}(\{1\})=2, \\
t_{1,2}\left(\iota_{2}(\{\})\right) & =t_{1,2}(1)=(1,2)(1)=2, \\
\iota_{2}\left(\tau_{1,2}(\{1\})\right) & =\iota_{2}(\{\})=1, \\
t_{1,2}\left(\iota_{2}(\{1\})\right) & =t_{1,2}()=(1,2)(1)=1, \\
\iota_{2}\left(\tau_{1,2}(\{2\})\right) & =\iota_{2}(\{2\})=3, \\
t_{1,2}\left(\iota_{2}(\{2\})\right) & =t_{1,2}(3)=(1,2)(3)=3 .
\end{aligned}
$$

Hence $\iota_{2}\left(\tau_{1,2}(I)\right)=t_{1,2}\left(\iota_{n}(I)\right)$. It also follows that

$$
\begin{aligned}
\iota_{2}\left(\tau_{2,2}(\{\})\right) & =\iota_{2}(\{2\})=3, \\
t_{2,2}\left(\iota_{2}(\{\})\right) & =t_{2,2}(1)=(1,3)(1)=3, \\
\iota_{2}\left(\tau_{2,2}(\{1\})\right) & =\iota_{2}(\{1\})=2, \\
t_{2,2}\left(\iota_{2}(\{1\})\right) & =t_{1,2}(2)=(1,3)(2)=2, \\
\iota_{2}\left(\tau_{2,2}(\{2\})\right) & =\iota_{2}(\{\})=1, \\
t_{2,2}\left(\iota_{2}(\{2\})\right) & =t_{2,2}(3)=(1,3)(3)=1 .
\end{aligned}
$$

Hence $\iota_{2}\left(\tau_{2,2}(I)\right)=t_{2,2}\left(\iota_{n}(I)\right)$ for $I \in \mathcal{I}_{2}$.

By Examples 4.5 and 4.5, we have $\iota_{n} \circ \tau_{k, n}=t_{k, n} \circ \iota_{n}$ for $n=1,2$. For $n>2$, we have the following lemmas: 
LEMmA 4.7. Let $n>2$ and $k<n-1$. If $\iota_{n-1} \circ \tau_{k, n-1}=t_{k, n-1} \circ \iota_{n-1}$, then $\iota_{n} \circ \tau_{k, n}=$ $t_{k, n} \circ \iota_{n}$.

Proof. First we consider the case where $\iota_{n}(I) \leqslant f_{n+1}$. In this case, $\mathcal{I}_{n-1}$ contains $I$. Hence we have $\tau_{k, n}(I)=\tau_{k, n-1}(I)$, which implies $\iota_{n}\left(\tau_{k, n}(I)\right)=\iota_{n}\left(\tau_{k, n-1}(I)\right)$. Since $\mathcal{I}_{n-1}$ contains $\tau_{k, n-1}(I)$, we have $\iota_{n}\left(\tau_{k, n-1}(I)\right)=\iota_{n-1}\left(\tau_{k, n-1}(I)\right)$. By assumption, we have

$$
\iota_{n-1}\left(\tau_{k, n-1}(I)\right)=t_{k, n-1}\left(\iota_{n-1}(I)\right)=t_{k, n-1}\left(\iota_{n}(I)\right) .
$$

Since $t_{k, n}=t_{k, n-1}, \iota_{n}\left(\tau_{k, n}(I)\right)=t_{k, n} \iota_{n}(I)$.

Next consider the case where $f_{n+1}<\iota_{n}(I)$. We show that $\iota_{n}\left(\tau_{k, n}(I)\right)$ equals

$$
\begin{aligned}
t_{k, n}\left(\iota_{n}(I)\right) & =t_{k, n-1} \hat{t}_{n} t_{k, n-1} \hat{t}_{n}^{-1}\left(\iota_{n}(I)\right) \\
& =\hat{t}_{n} t_{k, n-1} \hat{t}_{n}^{-1}\left(\iota_{n}(I)\right) \\
& =t_{k, n-1}\left(\iota_{n}(I)-f_{n+1}\right)+f_{n+1} .
\end{aligned}
$$

In this case we have $n \in I$. Hence $I^{\prime}=I \backslash\{n\} \in \mathcal{I}_{n-1}$ and $\iota_{n}(I)=\iota\left(I^{\prime}\right)+f_{n+1}$. It is easy to show that

Hence we have

$$
\tau_{k, n}(I)=\tau_{k, n-1}\left(I^{\prime}\right) \cup\{n\}
$$

$$
\begin{aligned}
\iota_{n}\left(\tau_{k, n}(I)\right) & =\iota_{n}\left(\tau_{k, n-1}\left(I^{\prime}\right) \cup\{n\}\right) \\
& =\iota_{n}\left(\tau_{k, n-1}\left(I^{\prime}\right)\right)+f_{n+1} \\
& =\iota_{n-1}\left(\tau_{k, n-1}\left(I^{\prime}\right)\right)+f_{n+1} .
\end{aligned}
$$

By assumption, we have $\iota_{n-1}\left(\tau_{k, n-1}\left(I^{\prime}\right)\right)=t_{k, n-1}\left(\iota_{n-1}\left(\left(I^{\prime}\right)\right)\right.$. Hence we have

$$
\begin{aligned}
\iota_{n}\left(\tau_{k, n}(I)\right) & =\iota_{n-1}\left(\tau_{k, n-1}\left(I^{\prime}\right)\right)+f_{n+1} \\
& =t_{k, n-1}\left(\iota_{n-1}\left(\left(I^{\prime}\right)\right)+f_{n+1}\right. \\
& =t_{k, n-1}\left(\iota_{n-1}\left((I)-f_{n+1}\right)+f_{n+1} .\right.
\end{aligned}
$$

LEMMA 4.8. Let $n>2$. If $\iota_{n-1} \circ \tau_{n-1, n-1}=t_{n-1, n-1} \circ \iota_{n-1}$, then $\iota_{n} \circ \tau_{n-1, n}=$ $t_{n-1, n} \circ \iota_{n}$.

Proof. First consider the case where $\iota_{n}(I) \leqslant f_{n+1}$. In this case, we have $I \in$ $\mathcal{I}_{n-1}$. Hence we have $\tau_{n-1, n}(I)=\tau_{n-1, n-1}(I)$, which implies $\iota_{n}\left(\tau_{n-1, n}(I)\right)=$ $\iota_{n}\left(\tau_{n-1, n-1}(I)\right)$. Since $\tau_{n-1, n-1}(I) \in \mathcal{I}_{n-1}$, we have

$$
\iota_{n}\left(\tau_{n-1, n-1}(I)\right)=\iota_{n-1}\left(\tau_{n-1, n-1}(I)\right) .
$$

Hence we have $\iota_{n-1}\left(\tau_{n-1, n-1}(I)\right)=t_{n-1, n-1}\left(\iota_{n-1}(I)\right)=t_{n-1, n-1}\left(\iota_{n}(I)\right)$. Since $t_{n-1, n}=t_{n-1, n-1}$, we have $\iota_{n}\left(\tau_{n-1, n}(I)\right)=t_{n-1, n}\left(\iota_{n}(I)\right)$.

Next we consider the case where $f_{n+1}<\iota_{n}(I)$. We show that $\iota_{n}\left(\tau_{n-1, n}(I)\right)$ equals $\hat{t}_{n-1}\left(\iota_{n}(I)\right)=\iota_{n}(I)$. In this case, $I$ contains $n$. Hence it follows that $\tau_{n-1, n}(I)=I$ and that $\iota_{n}\left(\tau_{n-1, n}(I)\right)=\iota_{n}(I)$.

LEMMA 4.9. We have $\iota_{n} \circ \tau_{n, n}=t_{n, n} \circ \iota_{n}$ for $n>2$.

Proof. First we consider the case where $\iota_{n}(I) \leqslant f_{n}$. We show that $\iota_{n}\left(\tau_{n, n}(I)\right)$ equals $\hat{t}_{n}\left(\iota_{n}(I)\right)=\iota_{n}(I)+f_{n+1}$. In this case, we have $I \in \mathcal{I}_{n-2}$, which implies $I$ does not contain $n-1, n$. Hence $\tau_{n, n}(I)=I \cup\{n\}$. Since $\iota_{n}(I \cup\{n\})=\iota_{n}(I)+f_{n+1}$ for $I \in \mathcal{I}_{n-2}$, it follows that $\iota_{n}\left(\tau_{n, n}(I)\right)=\iota_{n}(I)+f_{n+1}$.

Next we consider the case where $f_{n}<\iota_{n}(I) \leqslant f_{n+1}$. We show that $\iota_{n}\left(\tau_{n, n}(I)\right)$ equals $\hat{t}_{n}\left(\iota_{n}(I)\right)=\iota_{n}(I)$. In this case, we have $I \in \hat{\mathcal{I}}_{n-3}$, which implies $I$ contains $n-1$. Hence $\tau_{n, n}(I)=I$ and $\iota_{n}\left(\tau_{n, n}(I)\right)=\iota_{n}(I)$.

Finaly we consider the case where $f_{n+1}<\iota_{n}(I) \leqslant f_{n+2}$. We show that $\iota_{n}\left(\tau_{n, n}(I)\right)$ equals $\hat{t}_{n}\left(\iota_{n}(I)\right)=\iota_{n}(I)-f_{n+1}$. In this case, we have $I \in \hat{\mathcal{I}}_{n-2}$, which implies $I$ 
contains $n$. Hence $\tau_{n, n}(I)=I \backslash\{n\}$. Since $\iota_{n}(I \cup\{n\})=\iota_{n}(I)+f_{n+1}$ for $I \in \mathcal{I}_{n-2}$, it follows that $\iota_{n}\left(\tau_{n, n}(I)\right)=\iota_{n}(I)-f_{n+1}$.

Lemmas 4.7, 4.8 and 4.9 imply the following lemma:

LEMMA 4.10. If $n>2$ and $\iota_{n-1} \circ \tau_{k, n-1}=t_{k, n-1} \circ \iota_{n-1}$, then $\iota_{n} \circ \tau_{k, n}=t_{k, n} \circ \iota_{n}$.

Therefore, by induction on $n$, we have $\iota_{n} \circ \tau_{k, n}=t_{k, n} \circ \iota_{n}$ for $n \geqslant 1$.

Since $\iota_{n}$ induces a bijection between $\mathcal{I}_{n}$ and $F_{n+2}$, the bijective map

$$
\begin{aligned}
\left\{\tau_{1, n}, \ldots, \tau_{n, n}\right\} & \rightarrow G_{n} \\
\tau_{k, n} & \mapsto t_{k, n}
\end{aligned}
$$

induces the homomorphism from $\Gamma_{n}$ to $\left\langle G_{n}\right\rangle$ which preserves the actions. Since $G_{n}$ generates the $f_{n+2}$-th symmetric group $\mathfrak{S}_{f_{n+2}}$, the group $\Gamma_{n}$ is isomorphic to $\mathfrak{S}_{f_{n+2}}$. Hence $\left|\Gamma_{n}\right|=\left|\mathfrak{S}_{f_{n+2}}\right|=\left|\mathfrak{S}_{\mathcal{I}_{n}}\right|$. Since $\Gamma_{n} \subset \mathfrak{S}_{\mathcal{I}_{n}}$, we have Theorem 3.7.

Acknowledgements. The authors thank Matthew Macauley for the precious comments on results in the theory of automata related to this article. The authors thank anonymous referees for helpful suggestions.

\section{REFERENCES}

[1] Peter J. Cameron and Dmitri G. Fon-Der-Flaass, Orbits of antichains revisited, European J. Combin. 16 (1995), no. 6, 545-554

[2] Michael Joseph, Antichain toggling and rowmotion, Electron. J. Combin. 26 (2019), no. 1, Paper no. 1.29 (43 pages)

[3] Michael Joseph and Tom Roby, Toggling independent sets of a path graph, Electron. J. Combin. 25 (2018), no. 1, Paper no. 1.18 (31 pages).

[4] B Birational and noncommutative lifts of antichain toggling and rowmotion, Algebr. Comb. 3 (2020), no. 4, 955-984.

[5] Matthew Macauley, Jon McCammond, and Henning S. Mortveit, Dynamics groups of asynchronous cellular automata, J. Algebraic Combin. 33 (2011), no. 1, 11-35.

[6] Ville Salo, Universal gates with wires in a row, J. Algebraic Combin. (2021).

[7] Jessica Striker, The toggle group, homomesy, and the Razumov-Stroganov correspondence, Electron. J. Combin. 22 (2015), no. 2, Paper no. 2.57 (17 pages).

[8] Jessica Striker and Nathan Williams, Promotion and rowmotion, European J. Combin. 33 (2012), no. $8,1919-1942$.

Yasuhide Numata, Department of Mathematics, Shinshu University, Matsumoto, Japan E-mail : nu@math.shinshu-u.ac.jp

Yuiko Yamanouchi, Graduate School of Science and Technology, Shinshu University, Matsumoto, Japan

E-mail : 20ss118f@shinshu-u.ac.jp 